\title{
Quantum-Dot-Based Photon Emission and Media Conversion for Quantum Information Applications
}

\author{
H. Kumano, ${ }^{1,2}$ H. Nakajima, ${ }^{1}$ S. Ekuni, ${ }^{1}$ Y. Idutsu, ${ }^{1}$ \\ H. Sasakura, ${ }^{1,2}$ and I. Suemune ${ }^{1,2}$ \\ ${ }^{1}$ Research Institute for Electronic Science, Hokkaido University, Kita-21 Nishi-10, Kita-ku, \\ Sapporo 001-0021, Japan \\ ${ }^{2}$ CREST, Japan Science and Technology Agency, Kawaguchi 332-0012, Japan
}

Correspondence should be addressed to H. Kumano, kumano@es.hokudai.ac.jp

Received 18 September 2009; Revised 9 December 2009; Accepted 18 January 2010

Academic Editor: Shao-Ming Fei

Copyright (C) 2010 H. Kumano et al. This is an open access article distributed under the Creative Commons Attribution License, which permits unrestricted use, distribution, and reproduction in any medium, provided the original work is properly cited.

Single-photon as well as polarization-correlated photon pair emission from a single semiconductor quantum dots is demonstrated. Single photon generation and single photon-pair generation with little uncorrelated multiphoton emission and the feasibility of media conversion of the quantum states between photon polarization and electron spin are fundamental functions for quantum information applications. Mutual media conversion for the angular momentum between photon polarization and electron spin is also achieved with high fidelity via positively charged exciton state without external magnetic field. This is a clear indication that the coupling of photon polarizations and electron spins keeps secured during whole processes before photon emission. Possibility of a metal-embedded structure is demonstrated with the observation of drastic enhancement of excitation and/or collection efficiency of luminescence as well as clear antibunching of photons generated from a quantum dot.

\section{Introduction}

In order to fabricate quantum devices where the individual quantum particle plays an essential role in the device operations, a nonclassical guiding principle is required to describe the motion of individual quantum particle. Toward this direction, a field of quantum information science has made great stride in recent years [1]. In the quantum information science, information is coded on two-level quantum states called a quantum bit (qubit) based on a single quantum particle, and operations are carried out through an appropriate control of the qubit. Taking full advantage of some unique natures in quantum mechanics such as a superposition and a unitary transformation of states, these quantum operations on quantum devices are expected to show performances overwhelming those of classical devices. For instance, both 
absolutely secure communication with quantum key distribution (QKD) and much faster quantum computing than classical computers intrinsically owe to the quantum natures [2].

Since a photon is the fastest quantum object traveling with the speed of light and is robust to environmental fluctuations, the generation of single photons is fundamentally important for quantum information transport. They are generally called photon qubits, and on-demand generations of single photons and/or entangled photon pairs are highly required for realizing quantum information communication [3, 4]. Up to now, faint laser sources have been employed for the QKD experiments with optical fiber systems [5, 6] and also in free space [7-9]. However, inherent coherency of the laser source essentially leads to random photon numbers determined by the Poissonian statistics. This gives rise to inevitable probability of multiple photon generation and uncontrollable random timing of photon generation.

Single photon generation is possible by employing optical transitions in the two-level systems. A number of candidates have been studied up to now such as single atoms [10,11], single molecules [12], NV centers in diamond [13], gold nanoclusters [14], carbon nanotube [15] and semiconductor quantum dots (QDs) [16-25]. Among these systems, semiconductor QDs are regarded to be the most promising candidate from the viewpoint of realizing practical single photon diode sources [26]. For this purpose there remain several important issues to be solved such as the control of emission peaks and the reduction of uncorrelated background photon emission, as well as the elimination of fine-structure splitting (FSS) of exciton states [27], which lifts off the degeneracy to preserve the state superposition important for qubits.

In this article, single QD-based single-photon/photon-pair generation and mutual media conversion are reviewed and discussed. Experimental demonstration of single photon generation as well as polarization-correlated photon pair generation from semiconductor QDs will be presented. Angular momentum transfer from photon media to electron media and vice versa is essential for the future quantum information processing. Along this line, strongly preserved spin states of photo-excited exciton mediated by optical phonons is also demonstrated with a positively-charged exciton (trion) state. As a result, distinguishably high degree of circular polarization up to $\sim 0.85$ is achieved in the absence of external magnetic field, which yields high-fidelity media conversion. Possibility of a metallic cavity is demonstrated toward this direction.

\section{Experimental}

Two categories of QD samples, InAlAs and InAs QDs are investigated. The $\operatorname{In}_{0.75} \mathrm{Al}_{0.25} \mathrm{As}$ QDs sample was grown on a semi-insulating (001)-GaAs substrate by molecular-beam epitaxy. The QDs were prepared in Stranski-Krastanow (S-K) growth mode on $\mathrm{Al}_{0.3} \mathrm{Ga}_{0.7}$ As layers and were sandwiched with $\mathrm{Al}_{0.3} \mathrm{Ga}_{0.7}$ As layers. The topmost surface was terminated with a GaAs cap layer. After the growth, the sample was etched into mesa structures with diameters of $\sim 150 \mathrm{~nm}$ for isolating single QD from the dot ensemble with the density of around $5 \times 10^{10} \mathrm{dots} / \mathrm{cm}^{2}$. InAs QDs sample embedded in Niobium metal was also fabricated for enhancing the photon extraction efficiency. Details of sample preparation and the following processing methods are given elsewhere [28]. The optical characterization of the single QD was carried out with the experimental setup as depicted in Figure 1. A mode-locked Ti:Sapphire laser at the wavelength of $730 \mathrm{~nm}$ with the pulse width of $80 \mathrm{fs}$ and the pulse repetition frequency of $82 \mathrm{MHz}$ was used for pulsed excitations and a continuous-wave 


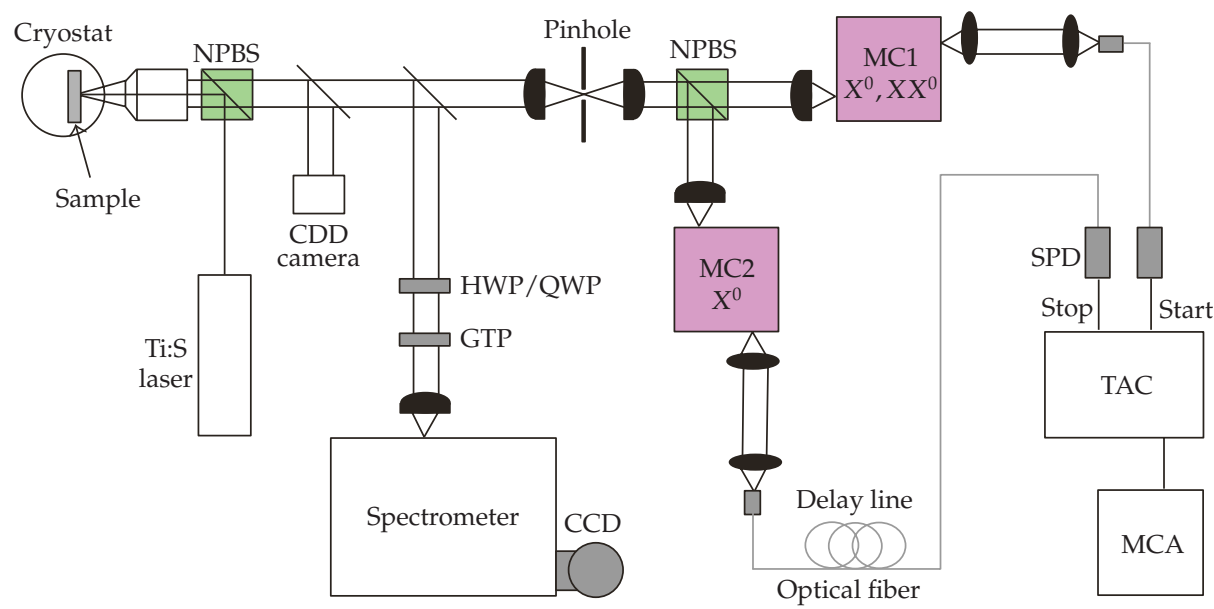

Figure 1: Overall experimental setup. CCD camera for imaging the sample surface, single/triple spectrometer equipped with CCD detector, and HBT setup for the second-order photon correlation measurements. Half wave plate (HWP), quarter wave plate (QWP), and Glan-Thomson polarizer (GTP) are used for polarization-dependent PL measurements. For photon correlation measurements, emitted photons are divided into two paths with non-polarized beam splitter (NPBS) and specific emission line is filtered by the monochromators (MC1 and MC2) set in each optical paths. Photons are detected by single photon detectors (SPD: EG and G single photon counting modules) through optical fibers. Generated electronic pulses at the SPDs are sent to a time-to-amplitude converter (TAC), and the temporal difference between the two outputs, $\tau=t_{\text {stop }}-t_{\text {start }}$, is accumulated to construct a histogram in multi-channel analyzer (MCA).

(CW) Ti:Sapphire laser was used for CW excitations. An objective lens with the numerical aperture (NA) of 0.42 focused the laser beam on one of the mesa structures and collected luminescence emitted from the mesa. Collected luminescence was dispersed by a 0.64-m triple monochromator and was introduced to a liquid-nitrogen cooled Si charge-coupleddevice detector. The PL polarization was analyzed in rectilinear $(\mathrm{H}, \mathrm{V})$ and / or circular $\left(\sigma^{+}\right.$, $\left.\sigma^{-}\right)$bases by a set of half/quarter-wave plates, where $\sigma^{+}\left(\sigma^{-}\right)$denotes the circular polarization with the helicity of $+1(-1)$. The overall system resolution was $4.5 \mu \mathrm{eV}$ and the measurements were done at $4 \mathrm{~K}-22 \mathrm{~K}$. For evaluating a photon statistics of emitted photons from a single QD, photon correlation measurements were carried out with the Hanbury-Brown and Twiss (HBT) setup [29] with auto- and cross-correlated configurations in order to test the singlephoton and correlated photon pair generation, respectively.

\section{Generation of Single Photons and Correlated Photon Pairs}

In the single QD spectroscopy, several emission lines were observed as shown in Figure 2. Neutral exciton $\left(X^{0} ; 778.143 \mathrm{~nm}\right)$, neutral biexciton $\left(X X^{0} ; 780.272 \mathrm{~nm}\right)$, positively charged exciton $\left(X^{+} ; 778.068 \mathrm{~nm}\right)$, and negatively charged exciton $\left(X^{-} ; 781.553 \mathrm{~nm}\right)$ are the dominant transition lines. The assignment of the above lines was done by a series of measurements on such as lifetimes, excitation power dependences, and polarization-selective PL measurements. It is noted that selective population to the specific emitting state is possible by selecting excitation power and/or energy as shown in the inset of Figure 2. This is also an important feature to implement the event-ready photon emission. 


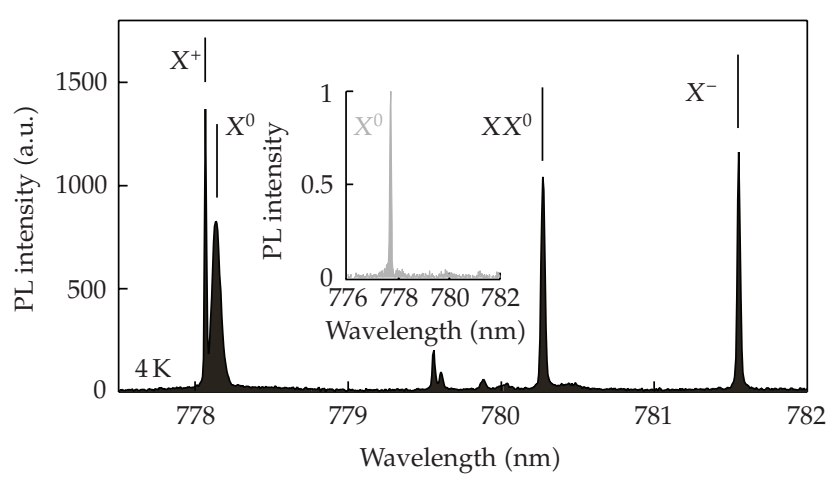

Figure 2: PL spectrum measured by single QD spectroscopy at $4 \mathrm{~K}$. Neutral exciton $\left(X^{0}\right)$, neutral biexciton $\left(X X^{0}\right)$, positively charged exciton $\left(X^{+}\right)$, and negatively charged exciton $\left(X^{-}\right)$dominate the spectrum. (Inset) PL spectrum under $X^{0}$ selective population with weak barrier layer excitation.

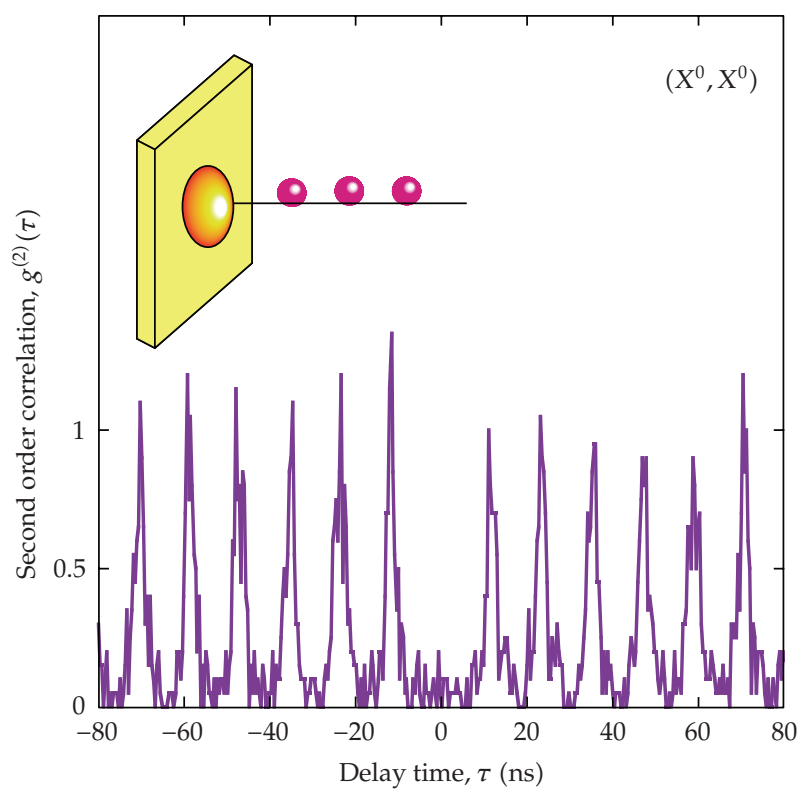

Figure 3: Result of second-order photon correlation measurement under autocorrelation configuration for the $X^{0}$ line. The Ti:Sapphire laser with the wavelength of $730 \mathrm{~nm}$ was used for the excitation. Observed peak separation of $\sim 12.2 \mathrm{~ns}$ corresponds to an excitation laser frequency of $82 \mathrm{MHz}$. Clear photon antibunching is observed at delay time $\tau=0$, which indicates at most single photon is emitted per single excitation event.

For a confirmation of the single photon emission per pulsed excitation, autocorrelation measurements under pulsed excitations were performed on the $X^{0}$ line. The excitation power was set to satisfy the condition on an average initial exciton number $\mu(0)<1$, and the resultant correlation function is illustrated in Figure 3. Observed peak period of $\sim 12.2 \mathrm{~ns}$ corresponds to the excitation pulse repetition of $82 \mathrm{MHz}$. Photon statistics can be evaluated by the second-order photon correlation function $\mathrm{g}^{(2)}(\tau)$, where $\tau$ is a delay time between the two paths. Strongly suppressed coincidence counts of $\mathrm{g}^{(2)}(0) \sim 0$ was observed, which indicates photon generation with highly suppressed multiphoton emission. 


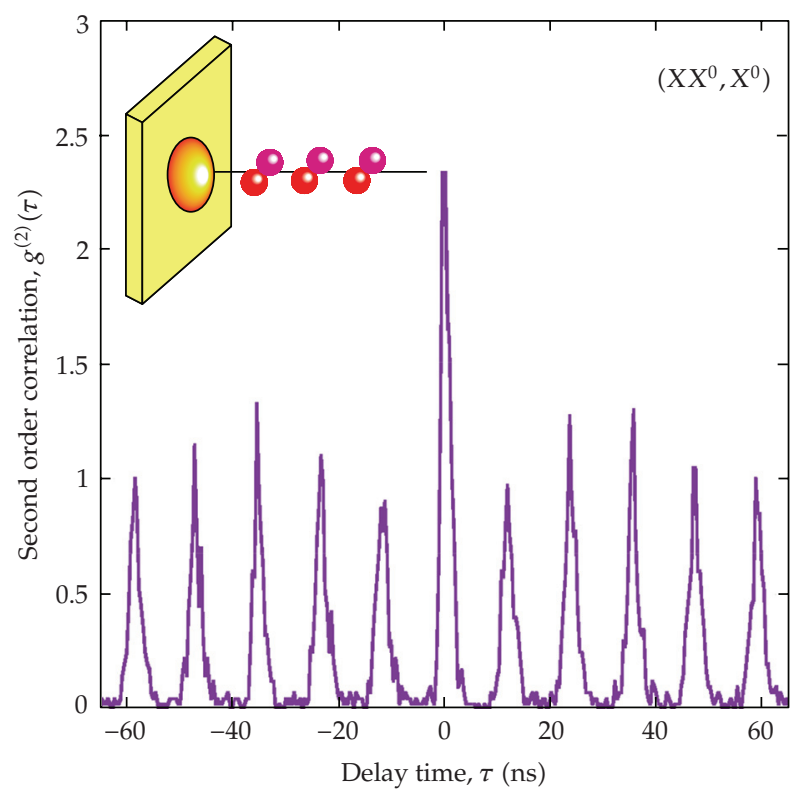

Figure 4: Second-order correlation function obtained with (start, stop) $=\left(X X^{0}, X^{0}\right)$ configuration. In this case, photon bunching behavior is clearly observed at delay time $\tau=0$. This indicates that the cascaded two-photon emission $X X^{0} \rightarrow X^{0}$ per single excitation event is taking place as illustrated by the inset.

When the two excitons (biexciton) with anti-parallel spins are populated in the QD, cascaded two photon emission via, $\left|X X^{0}\right\rangle \rightarrow\left|X^{0}\right\rangle \rightarrow|0\rangle$ is expected per single excitation event. Experimentally, this cascaded process is confirmed with the cross-correlation measurement under the (start, stop) $=\left(X X^{0}, X^{0}\right)$ configuration. Figure 4 shows the result of the cross-correlation measurement. In striking contrast to the auto-correlation in Figure 3, clear photon bunching behavior at $\tau=0$ was exhibited. This is a clear manifestation of two-photon states generation, in which the $X X^{0}$ and $X^{0}$ photons were emitted sequentially just after the excitation with laser pulses. Furthermore, polarization correlation between $X X^{0}$ and $X^{0}$ photons were observed (not shown), which indicates that the exciton spin flip time is longer than the exciton lifetime [30]. The $X X^{0}$ and $X^{0}$ lines investigated showed the FSS of $\sim 30 \mu \mathrm{eV}$. This is larger than the homogeneous broadening of $\sim 5 \mu \mathrm{eV}$ estimated by heterodyne four-wave mixing [31]. The large FSS leads to fast temporal evolution of the state vector as discussed in [32], which hinders us from reconstructing the incoming state as an entangled state after a series of measurements [33]. The FSS originates from crystallographic QD structural anisotropy naturally induced by the crystal-axis-dependent growth speed on semiconductor surfaces, and this tends to lift the spin degeneracy of the $X^{0}$ energy level via the electron-hole exchange interaction [27]. Very recently, however, generation of entangled photon pairs has been realized by optimizing growth or thermal annealing conditions [34-36] and by the post selection of generated photons [37].

\section{Conversion of Photon Polarization States and Exciton Spin States}

For the practical single photon sources especially for the QKD system, well-defined polarization of output photons is much preferable for subsequent coding process. Since the 


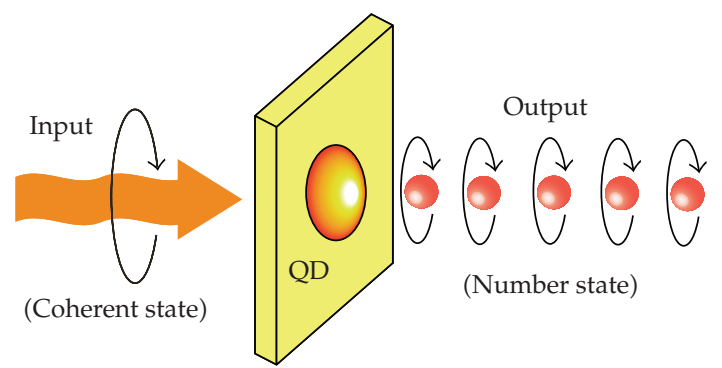

Figure 5: Schematic of polarization-preserved photon state converter based on a single quantum dot. Incident laser light is in the coherent state with the Poissonian photon number distribution. This photon statistics is converted ideally into the number state with deterministic and controllable output photon number per single excitation event.

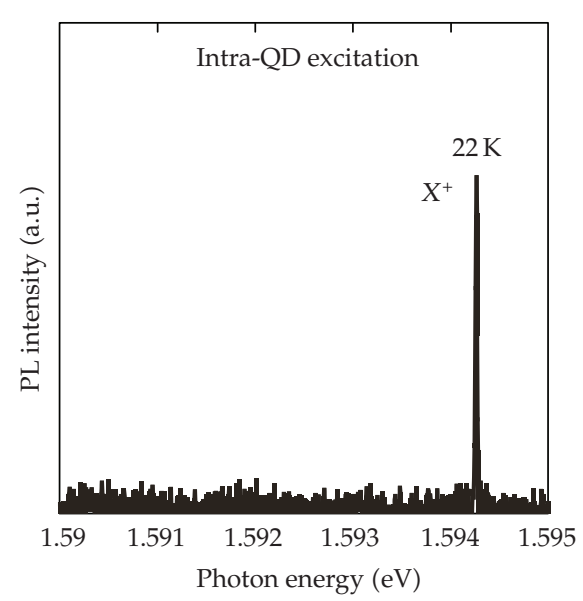

(a)

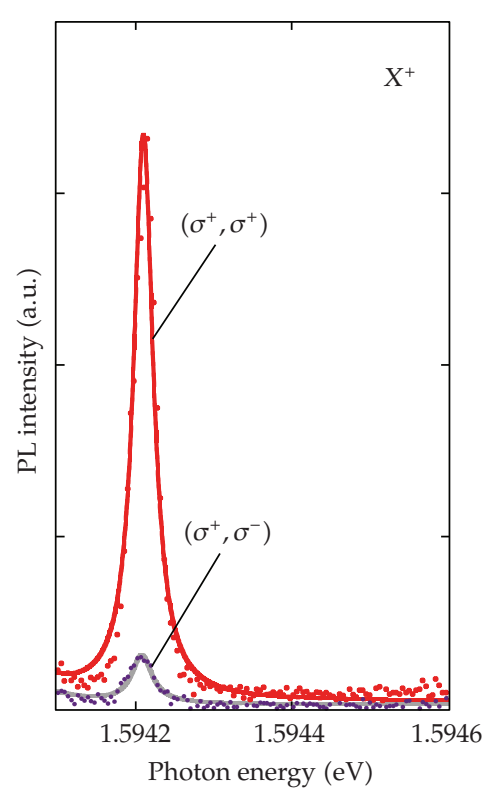

(b)

Figure 6: (a) $X^{+}$line selective population by intra-dot excitation. FSS is not observed since the two $X^{+}$states with their total spin of $\pm 1 / 2$ are degenerated. (b) Detection polarization-selective PL spectra of the $X^{+}$line measured under the $\sigma^{+}$excitation. Red curve is a result of co-circular $\left(\sigma^{+}\right)$detection, while the blue curve is a result of cross-circular $\left(\sigma^{-}\right)$detection. The degree of circular polarization defined by $\left(I_{\sigma^{+}}-I_{\sigma^{-}}\right) /\left(I_{\sigma^{+}}+I_{\sigma^{-}}\right)$ is as high as 0.85 without external magnetic field.

trion is a half-spin system, its ground states are degenerated due to Kramer's theorem in the absence of a magnetic field $[38,39]$, and the trion couples to circular-polarized photons guaranteed by a selection rule $[40,41]$. Therefore, the trion in a QD is quite promising for the high-fidelity photon state converter, where its spin state formed by the circularlypolarized excitation in coherent state will be ideally transferred into photons in number state with the incident polarization being preserved as illustrated in Figure 5. As shown in Figure 6(a), selective population of the $X^{+}$line is possible by intra-dot excitation. Figure $6(\mathrm{~b})$ shows the polarization-selective PL spectra of the $X^{+}$emission measured with circular basis 


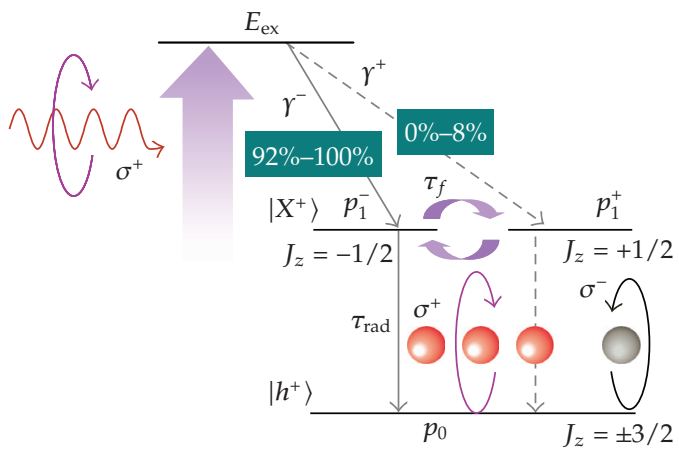

Figure 7: Schematic summary of the conversion process from incident coherent light (wavy arrow) to electron spins and further conversion to the single photon output. Polarization of photon is well preserved in the generated positive trion spins under the quasi-resonant excitation. The spin flip between the trion states with the opposite spin alignments will limit the spin preservation, but this influence is much reduced in the present trion state. The estimated fidelity, corresponding to the DCP of 0.85 , is as high as 0.96 .

under $\sigma^{+}$excitation. Here, the excitation energy is tuned to the InAs longitudinal-optical (LO) phonon energy $(30.5 \mathrm{meV})$ above the $\mathrm{X}^{+}$transition energy. It is revealed that there is striking difference between PL intensities detected with cocircular $\left(\sigma^{+}, \sigma^{+}\right)$and crosscircular $\left(\sigma^{+}, \sigma^{-}\right)$configurations. The resultant degree of circular polarization (DCP) defined as $\left(I^{+}-I^{-}\right) /\left(I^{+}+I^{-}\right)$, where $I^{+}\left(I^{-}\right)$denotes the PL intensity measured in the $\sigma^{+}\left(\sigma^{-}\right)$ polarization, amounts to 0.85 without external magnetic fields $[42,43]$. The resultant fidelity $\left[I^{+} /\left(I^{+}+I^{-}\right)\right]^{1 / 2}$ becomes as high as 0.96 . (Fidelity between two probability distributions of input and output photon polarization, $p^{\text {in }}$ and $p^{\text {out }}$, resp., is defined as $\sum_{i=+,-} \sqrt{p_{i}^{\text {in }} \cdot p_{i}^{\text {out }}}$, where $p_{ \pm}^{\text {in }}\left(p_{ \pm}^{\text {out }}\right)$ denotes the probability detecting $\sigma^{ \pm}$polarizations by measuring input (output) photons. In the present case, $\left(p_{+}^{\text {in }}, p_{-}^{\text {in }}\right)=(1,0)$ holds.)

In order to elucidate the spin flip in the entire processes involved after photoexcitation, a rate equation analysis was performed. In this model, a quantum dot with a residual hole state $\left|h^{+}\right\rangle$characterized by the angular momentum projection $J_{z}= \pm 3 / 2$ was considered as depicted in Figure 7. Preferential virtual excitation of a pair of spin-down electron and spin-up hole by the $\sigma^{+}$excitation to the energy one LO-phonon above the $\mathrm{X}^{+}$state was dealt with, that is, optical-phonon assisted resonant absorption in a single QD. Doubly degenerate $X^{+}$states are characterized by the angular momentum projection $J_{z}= \pm 1 / 2$. Then, corresponding rate equations are given by

$$
\begin{aligned}
& \frac{\mathrm{d} p_{0}}{\mathrm{~d} t}=-\gamma^{+} G p_{0}-\gamma^{-} G p_{0}+\frac{p_{1}^{+}}{\tau_{\mathrm{rad}}}+\frac{p_{1}^{-}}{\tau_{\mathrm{rad}}}, \\
& \frac{\mathrm{d} p_{1}^{ \pm}}{\mathrm{d} t}=-\gamma^{ \pm} G p_{0}-\frac{p_{1}^{ \pm}}{\tau_{\mathrm{rad}}}-\frac{p_{1}^{ \pm}}{\tau_{f}}+\frac{p_{1}^{\mp}}{\tau_{f}},
\end{aligned}
$$

where $p_{0}$ is the probability of finding the system in the single hole states, $p_{1}^{-}\left(p_{1}^{+}\right)$is the probability finding in the trion $J_{z}=-1 / 2\left(J_{z}=+1 / 2\right)$ state with a spin-down (up) unpaired electron, respectively, $G$ is the excitation rate, $\tau_{\text {rad }}$ is the radiative recombination lifetime of the trion, $\tau_{f}$ is the spin flip time between the $J_{z}= \pm 1 / 2$ trion ground states, and $\gamma^{+}\left(\gamma^{-}\right)$is the 
spin flip (preservation) probability during energy relaxation under the $\sigma^{+}$excitation, which satisfies $\gamma^{+}+\gamma^{-}=1$. By solving the rate equations in steady-state condition, DCP is expressed as

$$
\mathrm{DCP}=\frac{\left(\gamma^{-}-\gamma^{+}\right)\left(\tau_{f} / \tau_{\mathrm{rad}}\right)}{\left(\tau_{f} / \tau_{\mathrm{rad}}+2\right)}
$$

The relation of $\tau_{f} / \tau_{\text {rad }}$ and $\gamma^{+}$which reproduce the experimentally observed DCP is obtained and the main points derived from this analysis are twofold: The first point is that the ratio of $\tau_{f} / \tau_{\text {rad }}$ should be larger than 11 . Since the radiative lifetime of the $X^{+}$state was measured to be $1.0 \mathrm{~ns}$ [30], this indicates the spin flip time is longer than $11 \mathrm{~ns}$. It is noted that the obtained ratio of $\tau_{f} / \tau_{\text {rad }}$ is larger than that of the neutral exciton of $\sim 3.6$ measured on the same QD reported in [30]. This is partly because the $e$ - $h$ exchange interaction does not work in the trions and thus the effective magnetic field is quenched [44]. The second point of the rate equation analysis is that the allowed range of the spin flip probability $\gamma^{+}$during energy relaxation is quite low. The insertion of infinity for the $\tau_{f} / \tau_{\text {rad }}$ ratio in (4.2) gives the maximum spin flip probabilities of 0.075 from the measured DCP of 0.85 . Therefore the spin flip probability $\gamma^{+}$for the optical phonon-resonant excitations is given by the ranges of 0 0.075 . The spin state of the $X^{+}$created with LO phonon-assisted resonant excitation is shown to be highly stabilized.

In contrast to the $\gamma^{+}$parameter, the $\tau_{f} / \tau_{\text {rad }}$ ratio is inherent to the spin-flip mechanisms working on the trion ground states after energy relaxation. Under the steady-state circularly polarized excitation, the nuclear magnetic field can be induced by spin-polarized electrons [45], which possibly modifies the $\tau_{f} / \tau_{\text {rad }}$ ratio. Actually, the Zeeman splitting of $\sim 7 \mu \mathrm{eV}$ was observed in the $X^{+}$emission line under the WL excitation, which corresponds to the magnetic field of $B_{\mathrm{N}} \sim 0.33 \mathrm{~T}$ estimated with the $\mathrm{g}$ factor of $\mathrm{g}_{\mathrm{e}}=-0.37$ [46]. In order to examine the influence of dynamic nuclear polarization (DNP) $[28,47]$ on the observed high spin stability without an external magnetic field, the polarization modulation measurement of the DCP was performed and the results are shown in Figure 8. In this experiment, alternate circularly polarized excitation was carried out using an electro-optic modulator and the modulation frequency was swept sequentially. In order to deduce the DCP under alternate circularly polarized excitation, asymmetric duty ratio was used, that is, duty ratio of $\sigma^{+}: \sigma^{-}=2: 1$ $(1: 2)$ for measuring PL intensity $I^{+}\left(I^{-}\right)$with $\sigma^{+}\left(\sigma^{-}\right)$detection. Resultant degree of circular polarization under the present asymmetric excitation duty ratio is defined as $\rho \equiv\left[\left(2 I^{+}+I^{-}\right)-\right.$ $\left.\left(I^{+}+2 I^{-}\right)\right] /\left[\left(2 I^{+}+I^{-}\right)+\left(I^{+}+2 I^{-}\right)\right]=\mathrm{DCP} / 3$. Accordingly, the DCP in Figure 8 corresponds to $3 \rho$. As the modulation frequency was increased, the DCP started to decrease at $\sim 3 \mathrm{kHz}$ and then reaches to the almost constant value above $20 \mathrm{kHz}$. This behavior was unaffected by the sweep direction. Above $20 \mathrm{kHz}$ where the DNP can no longer follow the modulation, the DCP still exhibits as high as 0.5 . This indicates that the DNP has only a subsidiary contribution on the observed high DCP.

For the practical single photon emitters, suppressing multiphoton emission and improving photon extraction efficiency is strongly demanded for lowering bit error rate and enhancing transmission distance [48]. For this purpose, we have tried to embed a mesa structure containing a single InAs QD in metal to guide upward photon propagation for higher coupling to the objective lens. Here we have selected Niobium $(\mathrm{Nb})$ for the future implementation of superconductor-based QD light-emitting diodes [49]. First, GaAs pillar structures including InAs QDs inside were fabricated with electron beam lithography and 


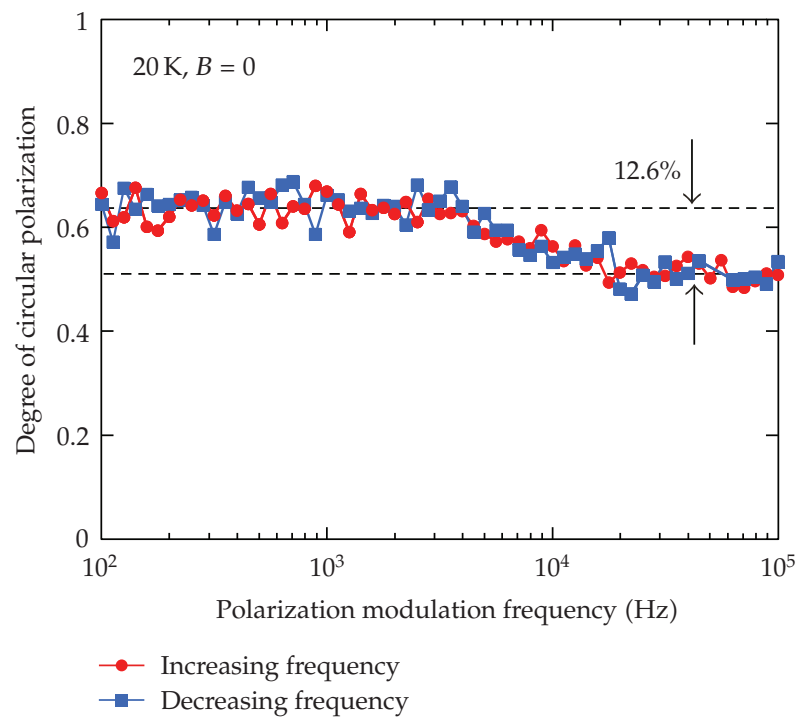

Figure 8: Polarization modulation frequency dependence of the DCP in $X^{+}$line. The effect of the DNP on stabilizing the spin state was limited and estimated to be $\sim 13 \%$ in terms of DCP.

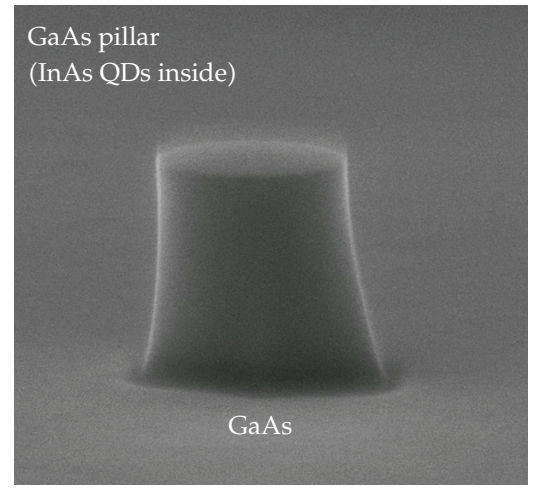

(a)

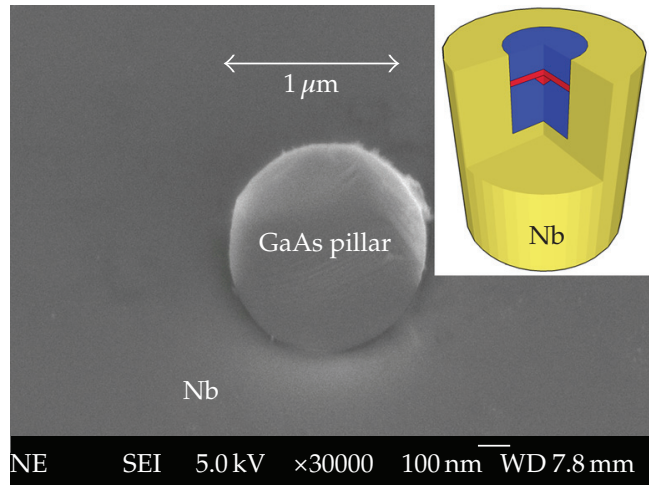

(b)

Figure 9: (a) SEM images of conventional GaAs pillar structure. InAs QDs are inside the pillar. (b) Top view of GaAs pillar after the $\mathrm{Nb}$ embedding. Schematic drawing of the $\mathrm{Nb}$-embedded pillar structure is shown in the inset.

dry etching. The scanning electron microscope (SEM) image of as-etched bare pillar structure is shown in Figure 9(a). Then the pillar structures were embedded with $\mathrm{Nb}$ with vacuum deposition equipment and the pillar samples were pasted on a supporting substrate with Indium. The pillars and deposited metal layers were transferred to the supporting substrate by removing the GaAs substrate of the pillar sample. The SEM image of the embedded pillar structures was observed in Figure 9(b) with a schematic drawing of the structure (inset). For the two samples, preliminary characterization was carried out under $\mathrm{CW}$ excitation. By isolating single QD, sharp emission lines with their transition energies of $\sim 1.34 \mathrm{eV}$ at $20 \mathrm{~K}$ are observed. The pillar diameter dependence of the square root of PL integrated intensity is investigated for both normal and embedded pillar structures and the results 


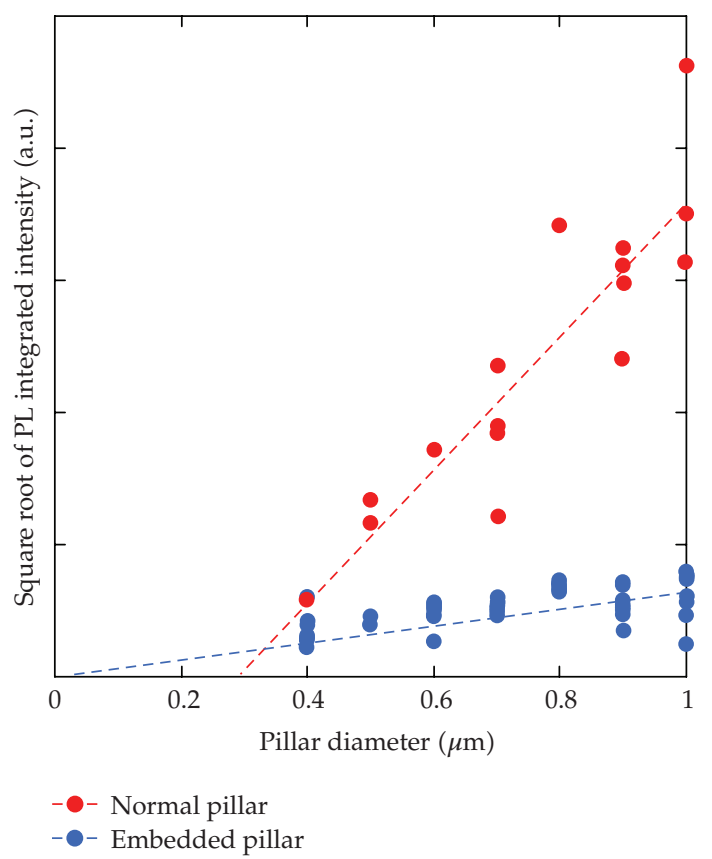

Figure 10: The pillar diameter dependence of the square root of PL integrated intensity. The fitting results by the least-square method are shown with the dashed line. The embedded pillar shows the 6.4-times increase of the slope. PL intensity is proportional to the luminescence area under the constant QD areal density. Thus the square root of PL integration intensity depends linearly on the pillar diameter. PL intensity is proportional to excitation rate $(G)$, internal quantum efficiency, number of QDs, light extraction efficiency $(C)$, and pillar cross section. Therefore, assuming the identical internal quantum efficiency for both structures, the fitted slope is proportional to $\sqrt{C G}$, which reveals $40\left(=6.4^{2}\right)$-times enhancement of $C G$, that is, the photon extraction efficiency including that of the excitation efficiency. In the $\mathrm{Nb}$ embedded structure, $0.3 \mu \mathrm{m}$ shifts from the origin is observed. This suggests formation of optically inactive region about $0.15 \mu \mathrm{m}$ from the $\mathrm{Nb}$ surface.

are compared in Figure 10. The embedded pillar shows the 6.4-times increase of the slope, which indicates $40\left(=6.4^{2}\right)$-times enhancement of the photon extraction efficiency including that of the excitation efficiency [50]. Second-order photon correlation function $\mathrm{g}^{(2)}(\tau)$ was measured and the result is displayed in Figure 11. In this measurement, LO-phonon resonant excitation was adopted because the quasiresonant excitation to the exciton ground level is advantageous from a viewpoint of suppressing multiphoton emission over the continuum state excitation [51,52]. The signal to background count ratio was approximately 150 . It is found that the correlation function at zero time delay $\mathrm{g}^{(2)}(0)$ is as low as 0.04 without background subtraction nor deconvolution with our system response function. This indicates that the metal-embedded pillar structure is quite promising for strongly suppressing the multiphoton emission, and realizing highly pure single photon emitters.

\section{Summary}

We have demonstrated the single-photon as well as polarization-correlated photon pair emission employing a single semiconductor quantum dot. Probability of the multiphoton emission is limited down to $\sim 1 / 25$ of the Poisson light. High-fidelity mutual media 


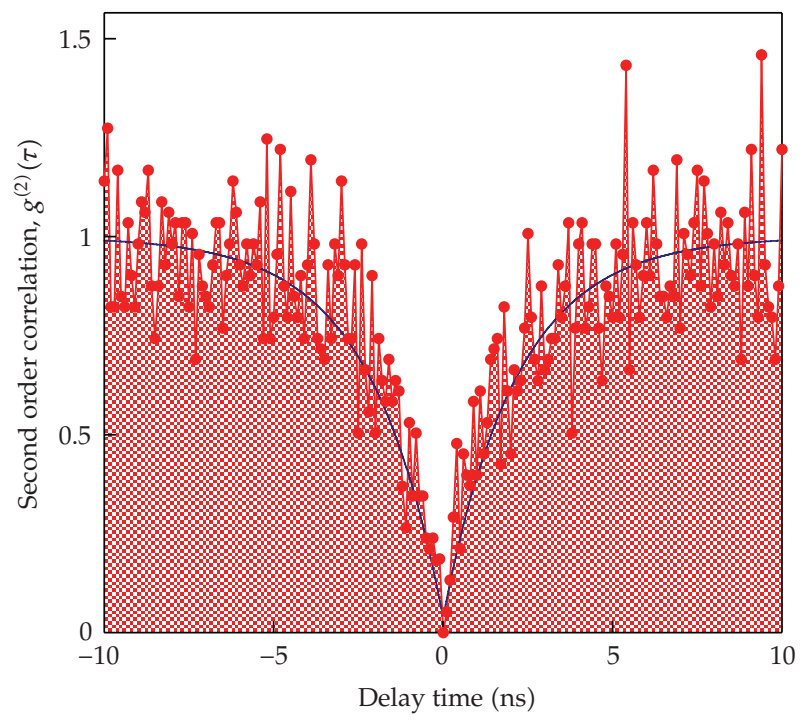

Figure 11: Second-order photon correlation function $\mathrm{g}^{(2)}(\tau)$ of single InAs QD embedded in Niobium metal. Blue line is a fitting result with a function $\mathrm{g}^{(2)}(\tau)=1-\operatorname{Aexp}(-\mathrm{B}|\tau|)$. Resultant $\mathrm{g}^{(2)}(0)$ is as low as $0.040 \pm 0.04$, and the time constant $B=2.17(\mathrm{~ns})$. This indicates that highly suppressed multiphoton emission is possible with the LO-phonon resonant excitation.

conversion for the angular momentum between photon polarization and electron spin is achieved via positively-charged exciton state without external magnetic field. The newly developed metal microcavity preserves high coupling efficiency for the interactions and conversions between photons and electron spins, which is quite preferable for the quantum information applications. The generation of photon qubits and conversion to spin qubits and vice versa based on solid states such as single quantum dots are still developing stage but show steady progress toward practical quantum information applications.

\section{Acknowledgments}

The authors would like to acknowledge Dr. H. Z. Song, S. Hirose, and M. Takatsu for the sample preparation. The authors are also grateful to Professor Adachi and Muto for their fruitful discussions. This work was supported in part by the Grant-in-Aid for Scientific Research (A) (2), no. 21246048, Young Scientists (A), no. 2168102009, and Hokkaido Innovation Through Nanotechnology Supports (HINTs) from the Ministry of Education, Culture, Sports, Science and Technology.

\section{References}

[1] I. Walmsley and P. Knight, "Quantum information science," Optics E Photonics News, vol. 13, no. 11, pp. 42-49, 2002.

[2] M. A. Nielsen and I. L. Chuang, Quantum Computation and Quantum Information, Cambridge University Press, Cambridge, UK, 2000.

[3] N. Gisin, G. Ribordy, W. Tittel, and H. Zbinden, “Quantum cryptography,” Reviews of Modern Physics, vol. 74, no. 1, pp. 145-195, 2002. 
[4] A. J. Shields, "Semiconductor quantum light sources," Nature Photonics, vol. 1, pp. 215-223, 2007.

[5] C. Gobby, Z. L. Yuan, and A. J. Shields, "Quantum key distribution over $122 \mathrm{~km}$ of standard telecom fiber," Applied Physics Letters, vol. 84, no. 19, pp. 3762-3764, 2004.

[6] H. Takesue, S. W. Nam, Q. Zhang, et al., "Quantum key distribution over a 40-dB channel loss using superconducting single-photon detectors," Nature Photonics, vol. 1, pp. 343-348, 2007.

[7] W. T. Buttler, R. J. Hughes, P. G. Kwiat, et al., "Free-space quantum-key distribution," Physical Review A, vol. 57, no. 4, pp. 2379-2382, 1998.

[8] C. Kurtsiefer, P. Zarda, M. Halder, et al., “Quantum cryptography: a step towards global key distribution," Nature, vol. 419, p. 450, 2002.

[9] R. J. Hughes, J. E. Nordholt, D. Derkacs, and C. G. Peterson, "Practical free-space quantum key distribution over $10 \mathrm{~km}$ in daylight and at night," New Journal of Physics, vol. 4, pp. 43.1-43.14, 2002.

[10] H. J. Carmichael, "Photon antibunching and squeezing for a single atom in a resonant cavity," Physical Review Letters, vol. 55, no. 25, pp. 2790-2793, 1985.

[11] J. McKeever, A. Boka, A. D. Boozer, et al., "Deterministic generation of single photons from one atom trapped in a cavity," Science, vol. 303, no. 5666, pp. 1992-1994, 2004.

[12] B. Lounis and W. E. Moerner, "Single photons on demand from a single molecule at room temperature," Nature, vol. 407, pp. 491-493, 2000.

[13] C. Kurtsiefer, S. Mayer, P. Zarda, and H. Weinfurter, "Stable solid-state source of single photons," Physical Review Letters, vol. 85, no. 2, pp. 290-293, 2000.

[14] J. I. Gonzalez, T.-H. Lee, M. D. Barnes, Y. Antoku, and R. M. Dickson, “Quantum mechanical singlegold-nanocluster electroluminescent light source at room temperature," Physical Review Letters, vol. 93, no. 14, Article ID 147402, 4 pages, 2004.

[15] A. Högele, C. Galland, M. Winger, and A. Imamoğlu, "Photon antibunching in the photoluminescence spectra of a single carbon nanotube," Physical Review Letters, vol. 100, no. 21, Article ID 217401, 2008.

[16] E. Moreau, I. Robert, J. M. Gérard, I. Abram, L. Manin, and V. Thierry-Mieg, "Single-mode solid-state single photon source based on isolated quantum dots in pillar microcavities," Applied Physics Letters, vol. 79, pp. 2865-2867, 2001.

[17] C. Becher, A. Kiraz, P. Michler, et al., "Nonclassical radiation from a single self-assembled InAs quantum dot," Physical Review B, vol. 63, no. 12, Article ID 121312(R), 4 pages, 2001.

[18] C. Santori, M. Pelton, G. Solomon, Y. Dale, and Y. Yamamoto, "Triggered single photons from a quantum dot," Physical Review Letters, vol. 86, no. 8, pp. 1502-1505, 2001.

[19] V. Zwiller, T. Aichele, W. Seifert, J. Persson, and O. Benson, "Generating visible single photons on demand with single InP quantum dots," Applied Physics Letters, vol. 82, pp. 1509-1511, 2003.

[20] X. Brokmann, E. Giacobino, M. Dahan, and J. P. Hermer, "Highly efficient triggered emission of single photons by colloidal CdSe/ZnS nanocrystals," Applied Physics Letters, vol. 85, p. 712, 2004.

[21] C. Couteau, S. Moehl, F. Tinjod, et al., "Correlated photon emission from a single 2-6 quantum dot," Applied Physics Letters, vol. 85, p. 6251, 2004.

[22] C. Santori, S. Gotzinger, Y. Yamamoto, S. Kako, K. Hoshino, and Y. Arakawa, "Photon correlation studies of single GaN quantum dots," Applied Physics Letters, vol. 87, Article ID 051916, 3 pages, 2005.

[23] K. Takemoto, Y. Sakuma, S. Hirose, et al., "Non-classical photon emission from a single InAs/InP quantum dot in the 1.3- $\mu \mathrm{m}$ optical-fiber band," Japanese Journal of Applied Physics, vol. 43, pp. L993L995, 2004.

[24] T. Miyazawa, K. Takemoto, Y. Sakuma, et al., "Single-photon generation in the 1.55- $\mu \mathrm{m}$ optical-fiber band from an InAs/InP quantum dot," Japanese Journal of Applied Physics, vol. 44, pp. L620-L622, 2005.

[25] S. Kimura, H. Kumano, M. Endo, et al., "Photon antibunching observed from an InAlAs single quantum dot," Japanese Journal of Applied Physics, vol. 44, pp. L793-L796, 2005.

[26] M. B. Ward, T. Farrow, P. See, et al., "Electrically driven telecommunication wavelength single-photon source," Applied Physics Letters, vol. 90, Article ID 063512, 3 pages, 2007.

[27] V. D. Kulakovskii, G. Bacher, R. Weignand, et al., "Fine structure of biexciton emission in symmetric and asymmetric CdSe/ZnSe single quantum dots," Physical Review Letters, vol. 82, no. 8, pp. 17801783, 1999.

[28] I. A. Merkulov, A. L. Efros, and M. Rosen, "Electron spin relaxation by nuclei in semiconductor quantum dots," Physical Review B, vol. 65, no. 20, Article ID 205309, 8 pages, 2002.

[29] R. Hanbury-Brown and R. Q. Twiss, "Correlation between photons in two coherent beams of light," Nature, vol. 177, pp. 27-29, 1956.

[30] H. Kumano, S. Kimura, M. Endo, et al., "Deterministic single-photon and polarization-correlated photon pair generations from a single InAlAs quantum dot," Optoelectronics, vol. 1, pp. 39-51, 2006. 
[31] T. Watanuki, S. Adachi, H. Sasakura, and S. Muto, "Long spin relaxation in self-assembled InAlAs quantum dots observed by heterodyne four-wave mixing," Applied Physics Letters, vol. 86, Article ID 063114, 3 pages, 2005.

[32] R. M. Stevenson, A. J. Hudson, A. J. Bennett, et al., "Evolution of entanglement between distinguishable light states," Physical Review Letters, vol. 101, no. 17, Article ID 170501, 4 pages, 2008.

[33] D. F. V. James, P. G. Kwiat, W. J. Munro, and A. G. White, "Measurement of qubits," Physical Review A, vol. 64, no. 5, Article ID 052312, 15 pages, 2001.

[34] C. A. Nicoll, C. L. Salter, R. M. Stevenson, et al., "MBE growth of $\operatorname{In}(\mathrm{Ga})$ As quantum dots for entangled light emission," Journal of Crystal Growth, vol. 311, no. 7, pp. 1811-1814, 2009.

[35] R. M. Stevenson, R. J. Young, P. Atkinson, K. Cooper, D. A. Ritchie, and A. J. Shields, "A semiconductor source of triggered entangledphoton pairs," Nature, vol. 439, no. 7073, pp. 179-182, 2006.

[36] R. J. Young, R. M. Stevenson, P. Atkinson, K. Cooper, D. A. Ritchie, and A. J. Shields, "Improved fidelity of triggered entangled photons from single quantum dots," New Journal of Physics, vol. 8, no. 2, article 29, 2006.

[37] N. Akopian, N. H. Lindner, E. Poem, Y. Berlatzky, J. Avron, and D. Gershoni, “Entangled photon pairs from semiconductor quantum dots," Physical Review Letters, vol. 96, no. 13, Article ID 130501, 4 pages, 2006.

[38] M. Bayer, G. Ortner, O. Stern, et al., "Fine structure of neutral and charged excitons in self-assembled In(Ga)As/(Al)GaAs quantum dots," Physical Review B, vol. 65, no. 19, Article ID 195315, 23 pages, 2002.

[39] I. A. Akimov, A. Hundt, T. Flissikowski, and F. Henneberger, "Fine structure of the trion triplet state in a single self-assembled semiconductor quantum dot," Applied Physics Letters, vol. 81, p. 4730, 2002.

[40] M. E. Ware, A. S. Bracker, E. Stinaff, D. Gammon, D. Gershoni, and V. L. Korenev, "Polarization spectroscopy of positive and negative trions in an InAs quantum dot," Physica E, vol. 26, no. 1-4, pp. 55-58, 2005.

[41] T. Flissikowski, I. A. Akimov, A. Hundt, and F. Henneberger, "Single-hole spin relaxation in a quantum dot," Physical Review B, vol. 68, no. 16, Article ID 161309(R), 4 pages, 2003.

[42] H. Kumano, H. Kobayashi, S. Ekuni, et al., "Excitonic spin-state preservation mediated by opticalphonon resonant excitation in a single quantum dot," Physical Review B, vol. 78, no. 8, Article ID 081306 (R), 4 pages, 2008.

[43] H. Kumano, S. Ekuni, and H. Kobayashi, "Spin-flip quenching in trion state mediated by optical phonons in a single quantum dot," Physica Status Solidi B, vol. 246, no. 4, pp. 775-778, 2009.

[44] G. V. Astakhov, A. V. Koudinov, K. V. Kavokin, et al., "Exciton spin decay modified by strong electronhole exchange interaction," Physical Review Letters, vol. 99, no. 1, Article ID 016601, 4 pages, 2007.

[45] C. W. Lai, P. Maletinsky, A. Badolato, and A. Imamoglu, "Knight-field-enabled nuclear spin polarization in aingle quantum dots," Physical Review Letters, vol. 96, no. 16, Article ID 167403, 2006.

[46] R. Kaji, S. Adachi, H. Sasakura, and S. Muto, "Precise measurements of electron and hole g factors of single quantum dots by using nuclear field," Applied Physics Letters, vol. 91, Article ID 261904, 2007.

[47] P.-F. Braun, X. Marie, L. Lombez, et al., "Direct observation of the electron spin relaxation induced by nuclei in quantum dots," Physical Review Letters, vol. 94, no. 11, Article ID 116601, 4 pages, 2005.

[48] E. Waks, C. Santori, and Y. Yamamoto, "Security aspects of quantum key distribution with subPoisson light," Physical Review A, vol. 66, no. 4, Article ID 042315, 7 pages, 2002.

[49] I. Suemune, T. Akazaki, K. Tanaka, et al., "Superconductor-based quantum-dot light-emitting diodes: role of cooper pairs in generating entangled photon pairs," Japanese Journal of Applied Physics, vol. 45, pp. 9264-9271, 2006.

[50] Y. Idutsu, M. Takada, S. Ito, et al., in Proceedings of the 51st Electronic Materials Conference (EMC '09), vol. B2, p. 15, University Park, Pennsylvania, Pa, USA, June 2009.

[51] A. Malko, M. H. Baier, K. F. Karlsson, E. Pelucchi, D. Y. Oberli, and E. Kapon, “Optimization of the efficiency of single-photon sources based on quantum dots under optical excitation," Applied Physics Letters, vol. 88, Article ID 081905, 3 pages, 2006.

[52] P. Ester, L. Lackmann, S. Michaelis de Vasconcellos, M. C. Hübner, and A. Zrenner, "Single photon emission based on coherent state preparation," Applied Physics Letters, vol. 91, Article ID 111110, 3 pages, 2007. 


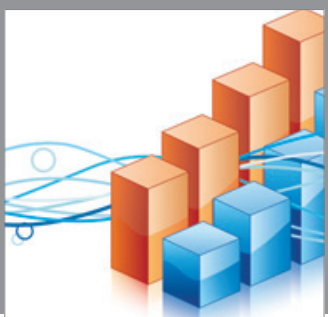

Advances in

Operations Research

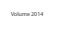

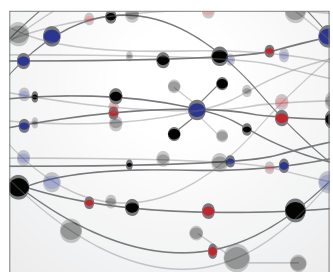

\section{The Scientific} World Journal
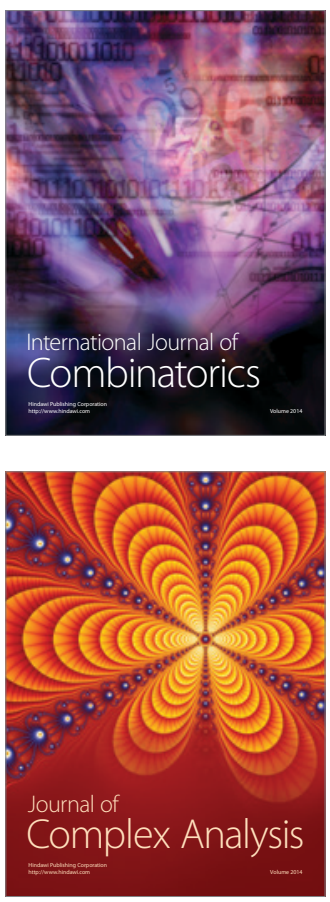

International Journal of

Mathematics and

Mathematical

Sciences
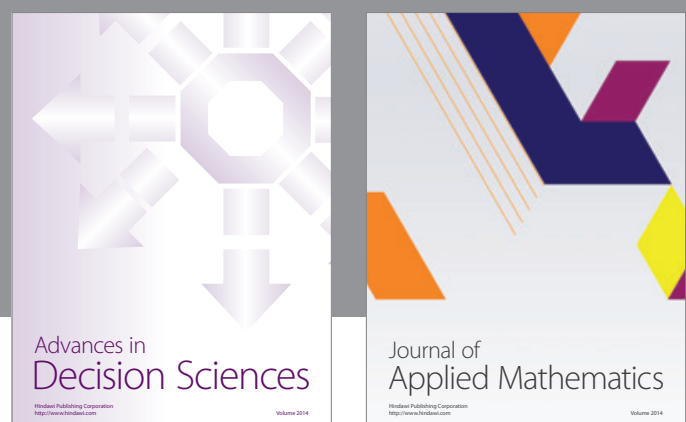

Journal of

Applied Mathematics
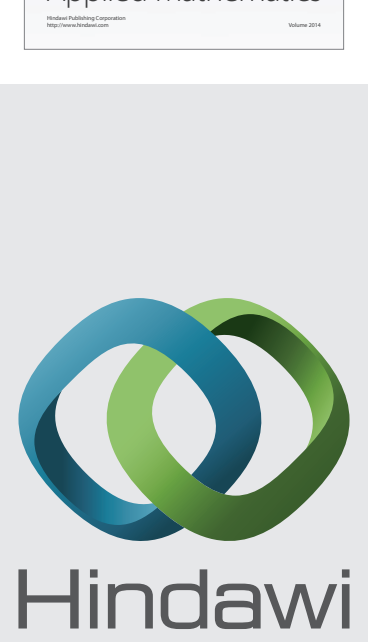

Submit your manuscripts at http://www.hindawi.com
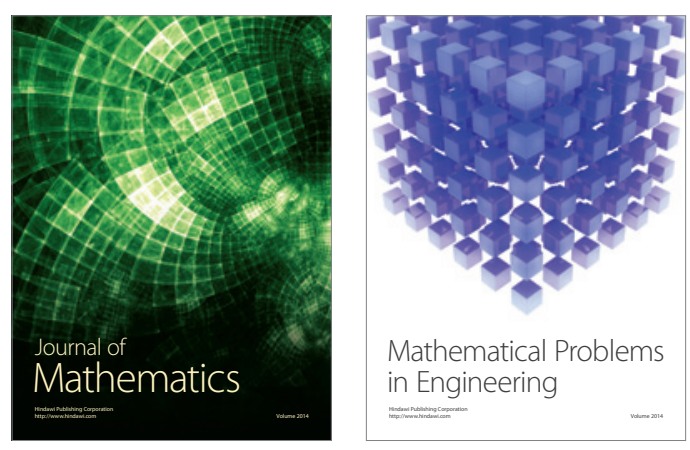

Mathematical Problems in Engineering
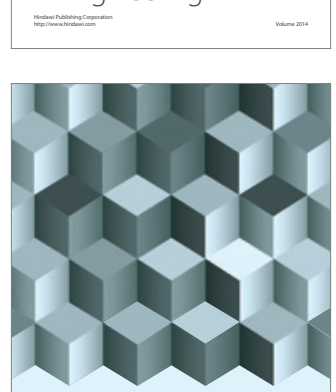

Journal of

Function Spaces
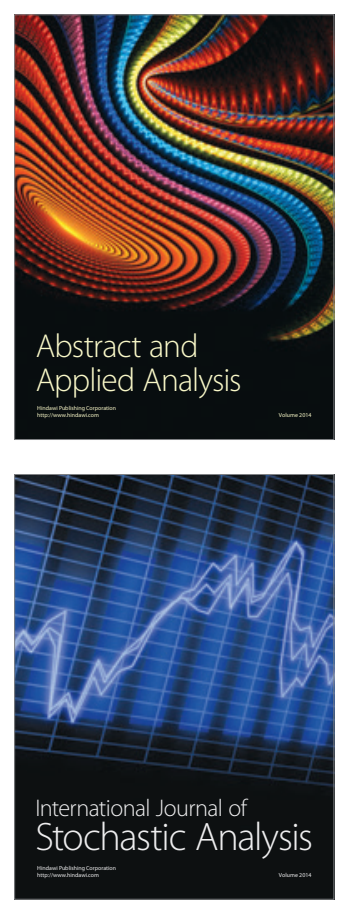

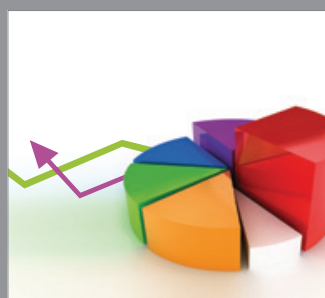

ournal of

Probability and Statistics

Promensencen
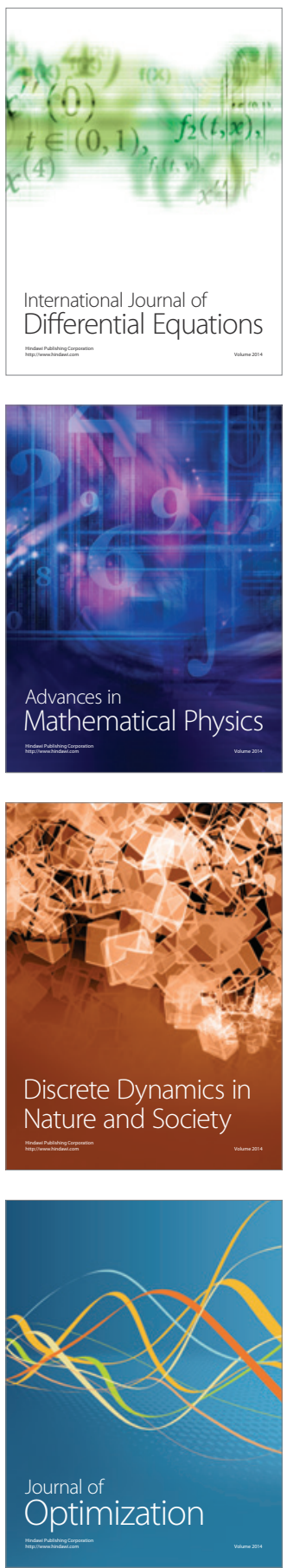